\title{
Review Paper \\ The Effectiveness of Rosa Canina Plant in Treatment of Some Diseases: A Brief Review
}

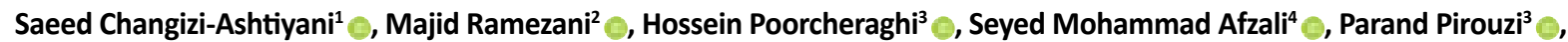 \\ Sina Atashi ${ }^{3}$, *Ali Zarei ${ }^{5}$
}

1. Department of Physiology, Faculty of Paramedicine, Arak University of Medical Sciences, Arak, Iran.

2. Department of Internal Medicine, Faculty of Medicine, Baqiyatallah University of Medical Sciences, Tehran, Iran.

3. Student Research Committee, Arak University of Medical Sciences, Arak, Iran.

4. Student Research Committee, Shiraz University of Medical Sciences, Shiraz, Iran.

5. Department of Physiology, Estahban School of Paramedical Estahban, Shiraz University of Medical Sciences, Shiraz, Iran.

\begin{tabular}{l|l}
$\begin{array}{l}\text { Use your device to scan } \\
\text { and read the article online }\end{array}$ & $\begin{array}{l}\text { Cftation: Changizi-Ashtiyani S, Ramezani M, Poorcheraghi H, Afzali SM, Pirouzi P, Atashi S, et al. [ The Effectiveness of } \\
\text { Rosa Canina Plant in Treatment of Some Diseases: A Brief Review (Persian)]. Journal of Arak University of Medical Sciences } \\
\text { (JAMS). 2019; 22(5):6-17. https://doi.org/10.32598/JAMS.22.5.6 }\end{array}$ \\
dofltps://doi.org/10.32598/JAMS.22.5.6
\end{tabular}

\section{(c) 0 (3)}

Article Info:

Received: 21 Nov 2018

Accepted: 15 May 2019

Available Online: 01 Dec 2019

Key words:

Rosa canina, Liver, Diabetes, Blood lipids, Cancer, Vitamin C

\section{A B STRACT}

Background and Aim Among the plants with multiple healing properties and a long and common history of use in Iranian traditional medicine, is dog-rose plant with the scientific name of "Rosa canina" that belongs to the Rosaceae family. This study aimed to review the effectiveness of Rosa canina in treatment of different body organs and tissues and emphasizes its therapeutic mechanism.

Methods \& Materials This is a review study. About 176 papers published from 2010 to 2018 were extracted from PubMed, Science Direct, Scopus, Google Scholar, Ovid, MagIran, and SID databases using related keywords and entered into study.

Ethical Considerations Ethical issues (including plagiarism, misconduct, data fabrication, falsification, double publication or submission, redundancy) have been completely observed by the authors.

Results Different studies had indicated the importance of Rosa canina as a medicinal plant protecting liver, lowering glucose and lipid levels, and having antioxidant and anti-inflammatory properties. The highest flavonoid activity in the Rosaceae family is related to Rosa canina. The use of herbal extracts in the treatment of common diseases, such as diabetes, fatty liver, urinary tract and Alzheimer's, has been recommended.

Conclusion High qualitative and quantitative diversity of effective ingredients, ease of access, absence of adverse effects of consumption and endemic nature of Rosa canina plant make it necessary to use it for the control and treatment of common human diseases.

\section{Extended Abstract}

\section{Introduction}

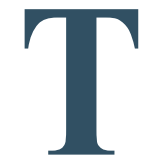

oday, with the increasing number and variety of chronic diseases, the use of chemical drugs to control them has increased. However, the adverse effects of using chemical drugs, tolerance over time, high costs, and uncertainty of treatment have made the tendency to use medicinal plants. In addition to reducing treatment costs, these herbs have had satisfactory results in many societies which Rosa Canina $\mathrm{L}$ is one of these plants $[2,3]$.

* Corresponding Author:

Ali Zarei, PhD.

Address: Department of Physiology, Estahban School of Paramedical Estahban, Shiraz University of Medical Sciences, Shiraz, Iran.

Tel: +98 (917) 1502855

E-mail: zarei.ali40@gmail.com 


\section{Materials and Methods}

The papers published in English and Persian were searched in PubMed, Science Direct, Scopus, Google Scholar, Ovid, Magiran, and SID databases by using the following keywords: Rosa canina, Disease, Antioxidant plants, Osteoarthritis, Immune system, Reperfusion injury.

\section{Results}

This plant contains valuable properties such as antioxidant activity, rich vitamins (especially vitamin C), and phenolic compounds [10]. Its phenolic compounds are capable of having a wide range of biochemical characteristics, including antimutagenic and anticarcinogenic properties [11]. Furthermore, ascorbic acid, as the main water-soluble antioxidant within the body, has anticarcinogenic and other biological properties $[12,13]$. Ascorbic acid has a significant regulatory function throughout the body because it is engaged in the synthesis of hormones, hormone-releasing factors, and neurotransmitters [15].

The protective effects of rose hip against ischemia-reperfusion induced renal functional and structural injuries that were confirmed in another animal study [4، 32]. The hepatoprotective effects of Rosa canina fruit extract have been documented in a rat model of carbon tetrachloride-induced hepatotoxicity, too $[29,30]$. More interestingly, hydroalcoholic extract of Rosa canina fruit decreased both urinary calcium contents and the size and number of calcium oxalate calculi in the kidneys in a rat model of experimentally induced nephrolithiasis [34].

\section{Discussion}

This review article results show that Rosa canina is high in antioxidant and anti-inflammatory properties due to its valuable content of vitamin $\mathrm{C}$, polyphenols, carotenoids. In addition, being native, inexpensive, affordable and, most importantly, low or with no side effects, has led the plant to be used as a new remedy in a large number of chronic diseases. The use of its aqueous extracts (in the form of demos and teas) has the ability to inhibit lipid peroxidation. In existing treatment protocols to improve or control the symptoms of many diseases such as disorders in liver, gastrointestinal tract, eyes, skin, and etc. Today, there is a need for deeper and wider research to revitalize the field of further understanding of the beneficial effects of this plant and its benefits, especially in the pharmaceutical aspects.

\section{Ethical Considerations}

\section{Compliance with ethical guidelines}

In this paper, the principles of publication ethics were considered.

\section{Funding}

This study received financial support from the Deputy for Research and Technology of Arak University of Medical Sciences.

\section{Authors' contributions}

Conceptualization, initial draft preparation, investigation, and editing: Saeed Changizi-Ashtiyani, Majid Ramezani and Ali Zarei; Investigation and editing: Hosein Poorcheraghi, Seyed Mohammad Afzali, Parand Pirouzi, and Sina Atashi.

\section{Conflicts of interest}

The author declared no competing interests

\section{Acknowledgements}

The authors would like to thank the Deputy for Research and Technology of Arak University of Medical Sciences for their support. 



\title{
بررسى اثربخشى تياه نسترن كوهى (Rosa Canina L) در برخى از بيمارىها (يك مطالعه مرورى)
}

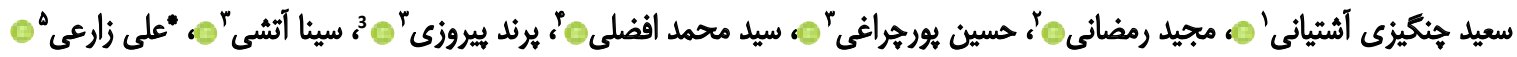

\author{
I. كروه فيزيولوزي، دانشكده يبرايزشكى، دانشكاه علوم يزشكى اراك، اراك، ايران.

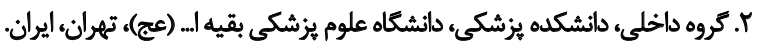

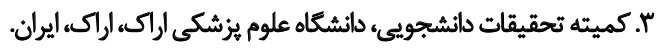

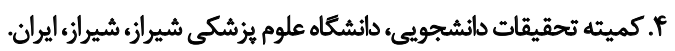 \\ هـ كروه فيزيولوزى، دانشكده ييرايزشكى استهبان، دانشعاه علوم يزشكى شيرازئ، شيراز، ايران.
}

\begin{abstract}
سيكين

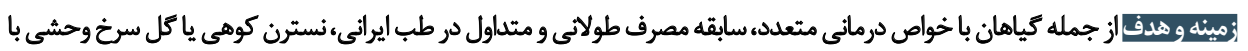

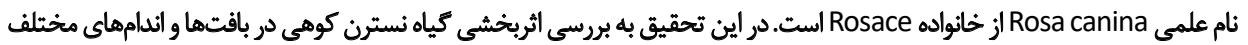

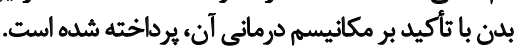

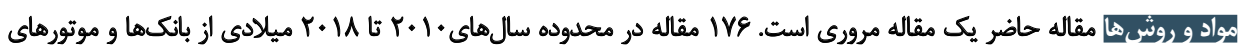

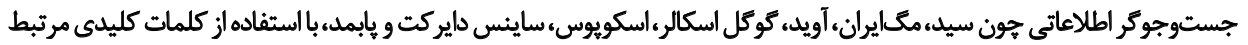

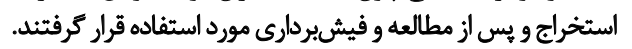

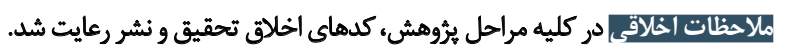

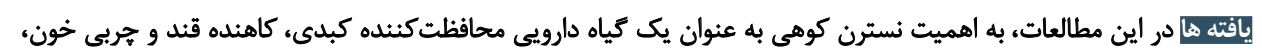

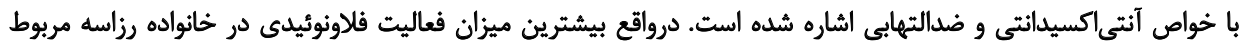

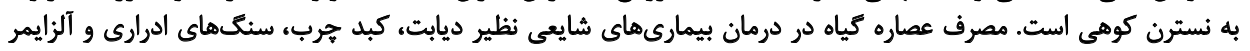
توصيه شده است.

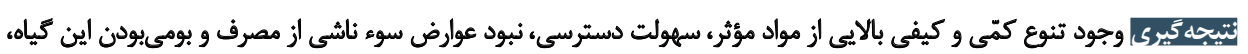

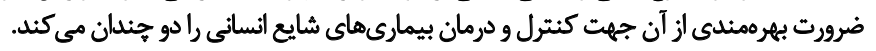

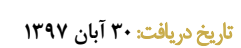

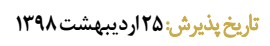

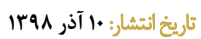

كليدواروها: نسترن كوهى، كبد، ديابت، جربى خون، كيد،

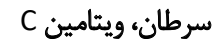

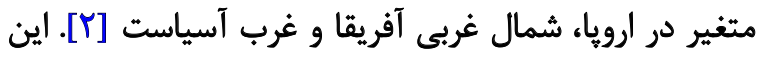

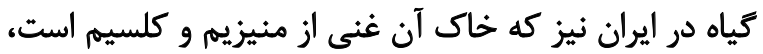

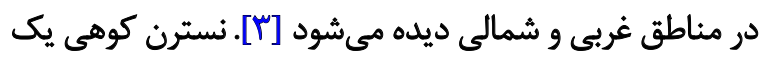

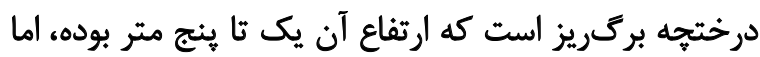

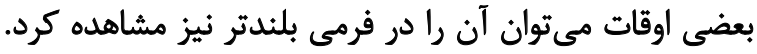

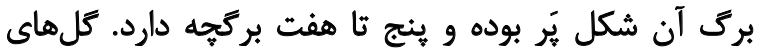

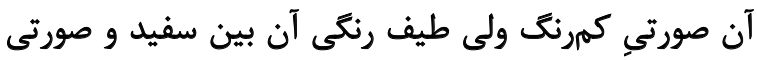

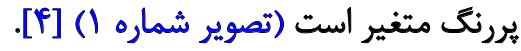
نيني الدر (A.D Yr-V9) اولين فردى است كه خواص دارويى

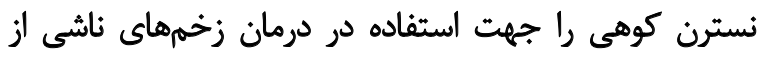

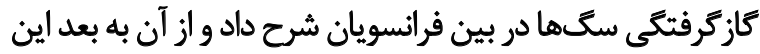

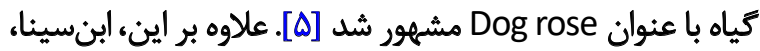

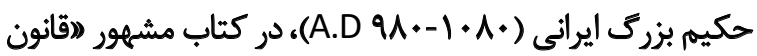

dalê

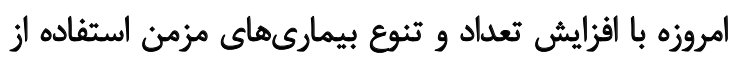

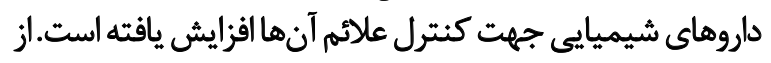

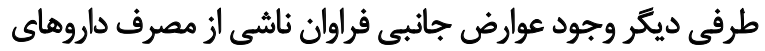

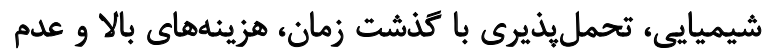

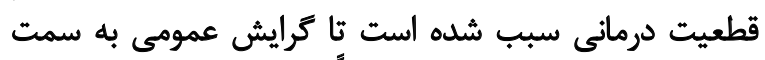

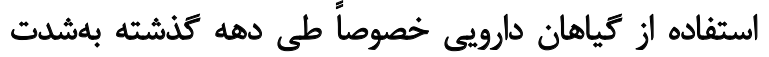

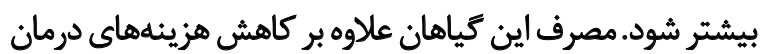

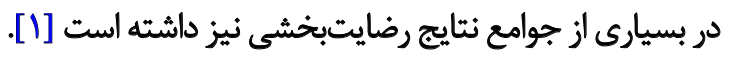

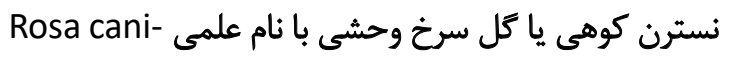

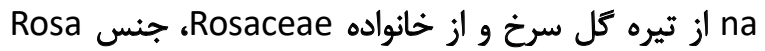

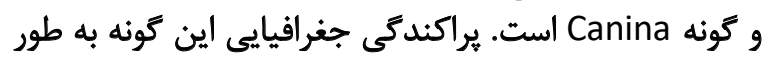

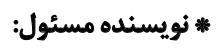

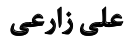

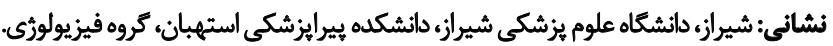

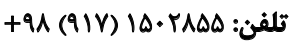
يست الكترونيكى: zarei.ali40@gmail.com 


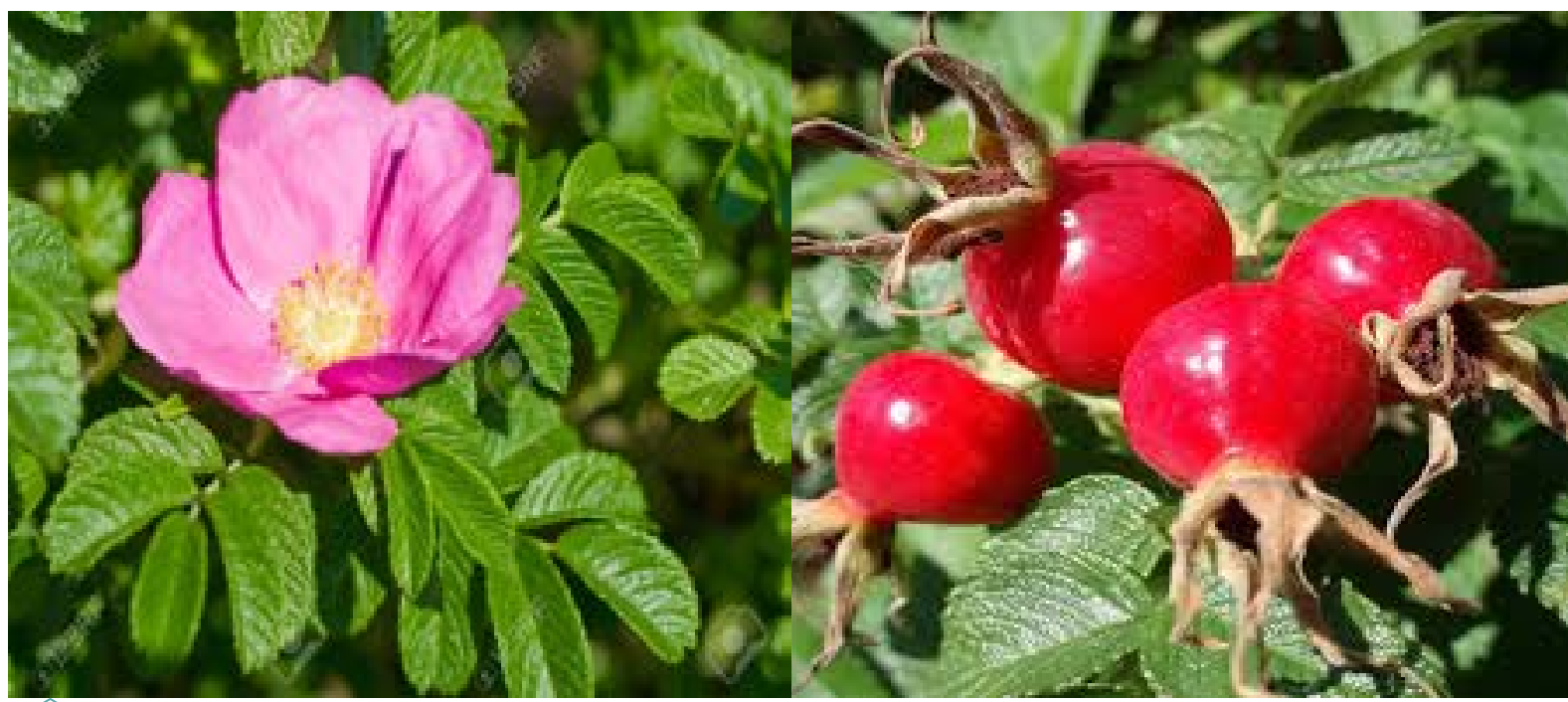

$\left(s^{m}\right)$

خواص خاص اين كياه به محققين و علاقهمندان جهت كنترل و درمان بيمارى هاى شايع و مزمن انسانى معرفى شود.

$$
\text { واش كار }
$$

مطالعه حاضر يك مقاله مرورى است. جستوجوى مقالات

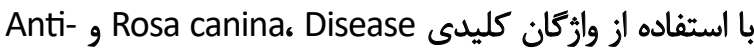
و Osteoarthritis Immune system , oxidant plants Diabetes geperfusion injury

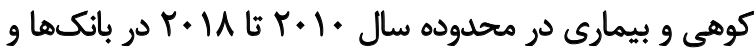

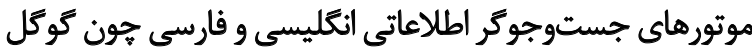

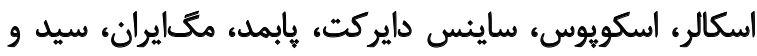

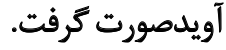

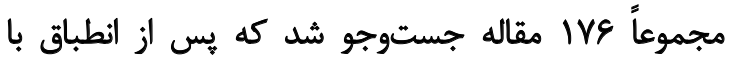

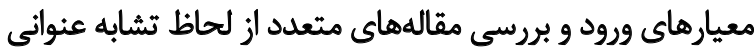

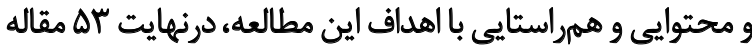

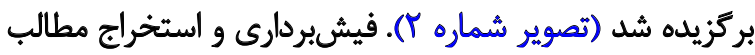

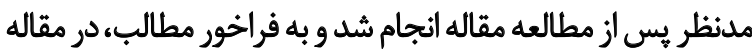

$$
\text { حاضر مورد استناد قرار كرفت. }
$$

در اين بخش اثرات دانه، برك و ميوه نسترن كوهى بر بيمارىها و بخشهاى مختلف مطرح مى شود.

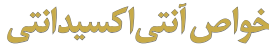

در مطالعات اخير، محققان توجه بسيارى به خواص

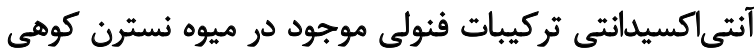

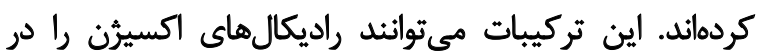

تصوير ا. ميوه و كل كياه نسترن كوهى (Rosa Canina)

در طبه) خود به كياه مذكور با عنوان علف سك جهت بهان بهبود

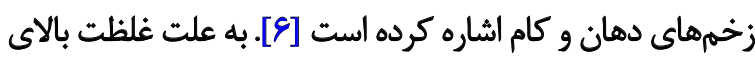

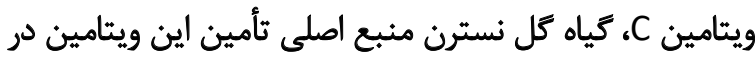

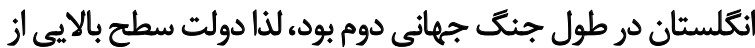
زمينها راجهت به كشت اين كياه اختصاص داد [V] بخشهاى مختلف اين كياه به طور سنتى براى درمان

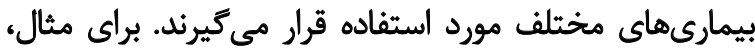

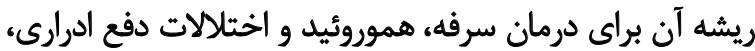

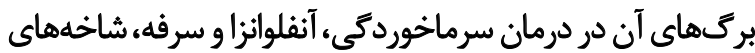

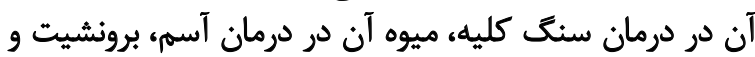

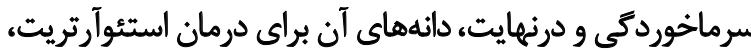

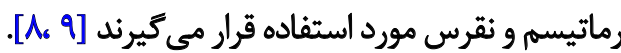
به طور سنتى، مرباو سوب اين كياه در كشورهاي اسكانديناوى

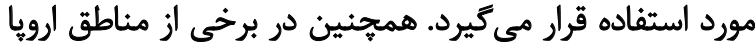

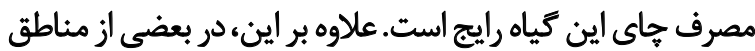

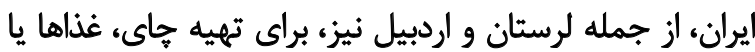

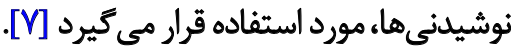
از جمله تركيبات مؤثر در ميوهنسترن كوهي ميى توان بهويتامين

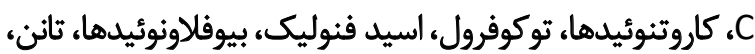

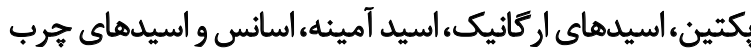

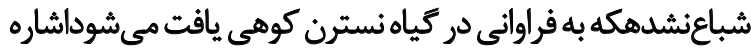

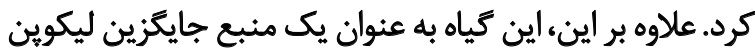

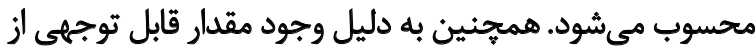

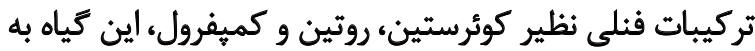

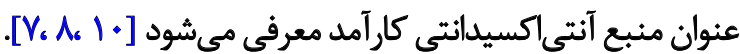

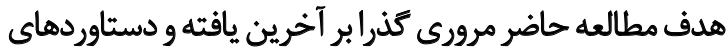

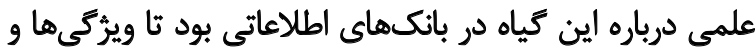




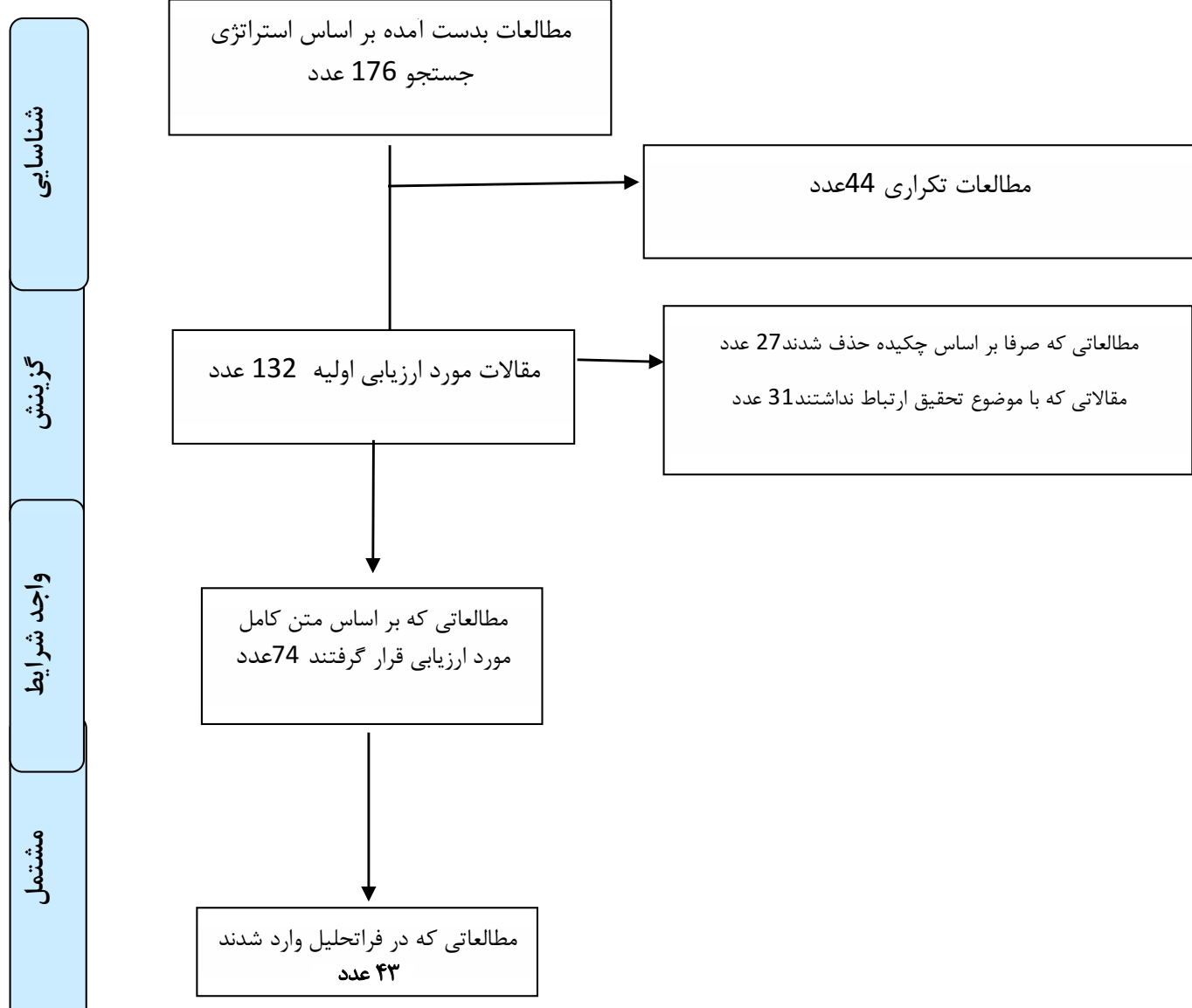

تصوير r. فلوجارت انتخاب و مداخله كل مقالات جستوجوشده در مطالعه

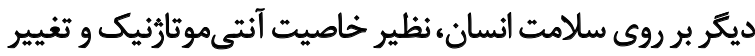

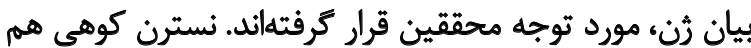

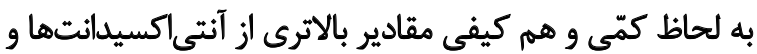

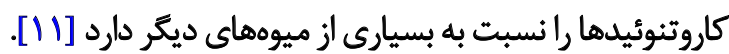

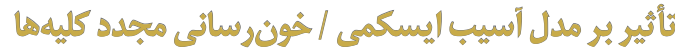

ايسكمى عامل مهم نارسايى حاد كليوى است كه با آغازكردن

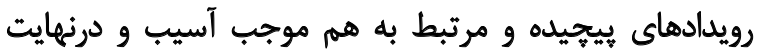
مرى سلولهاى كليه مى

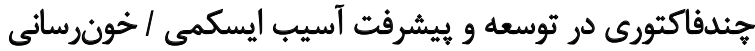

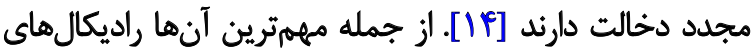

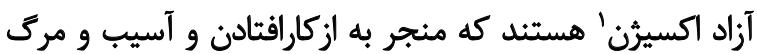

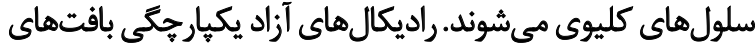

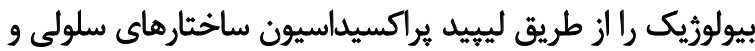

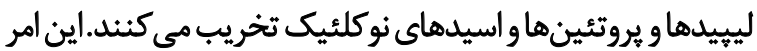

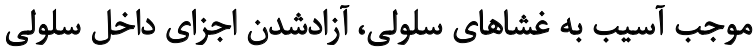

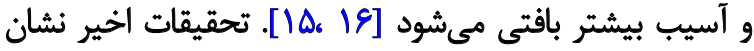

1. Reactive Oxygen Species (ROS)

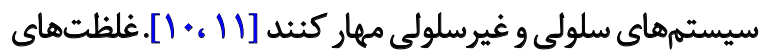

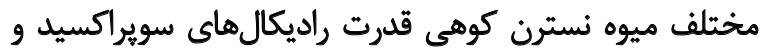

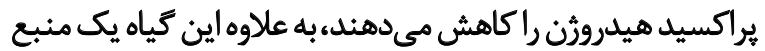

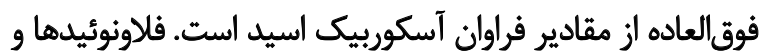

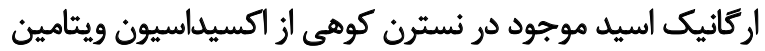

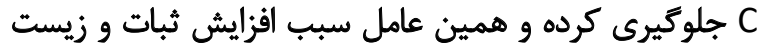

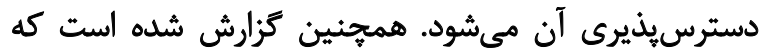

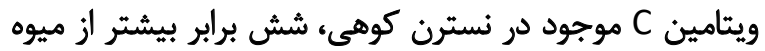

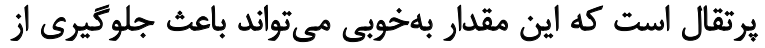
فعاليت آلفا آميلاز شود [1 آ]. در مطالعه ديكرى مشخص شد كه عصاره آبي و الكلى نسترن

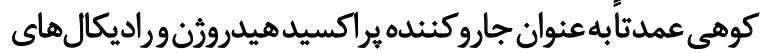

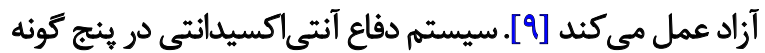

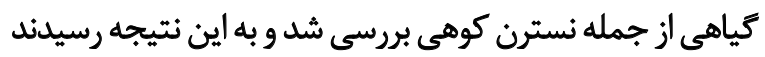

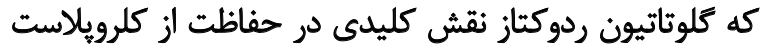

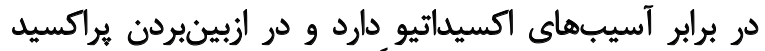

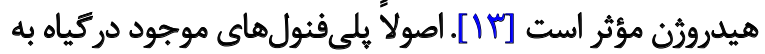
علت داشتن خواص آنتىاكسيدانتى و يرواكسيدانتى و اثرات مهرم 


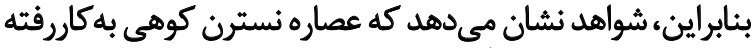

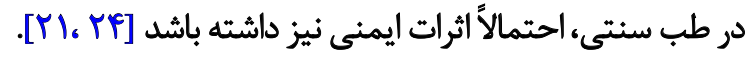
خواص ضدالتهابى اين تياه در مطالعات موجودات زنده

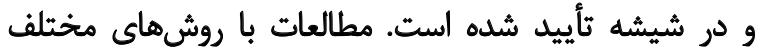

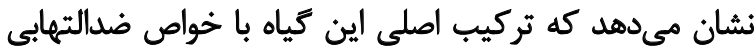

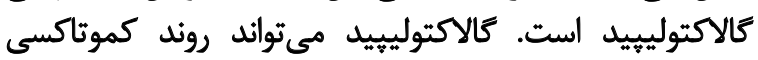

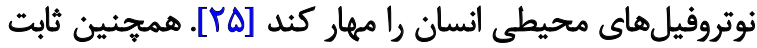

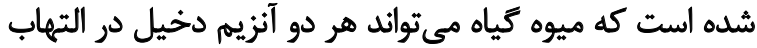

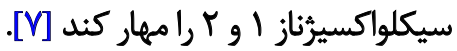

بتاسيتوسترول يك فيتوسترول موجود در كياه نسترن كوهى

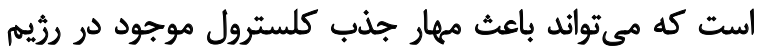

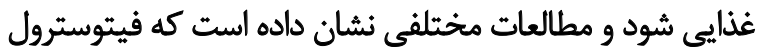

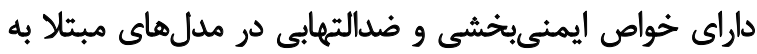

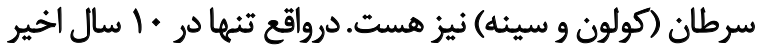

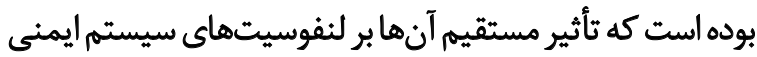

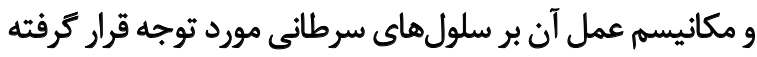

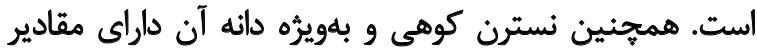

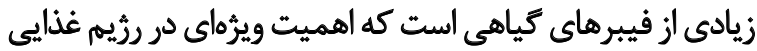

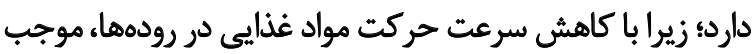

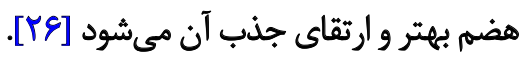

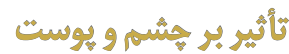

كياه نسترن كوهى حاوى مقاديرى كوئرستين، به عنوان يك إني

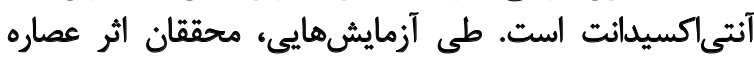

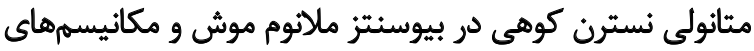

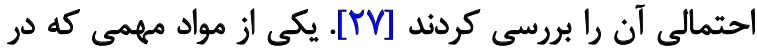

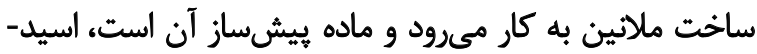

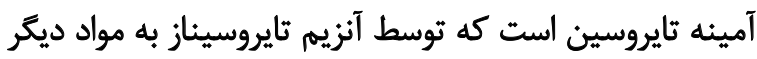

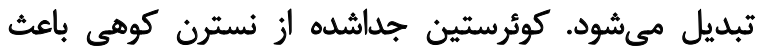

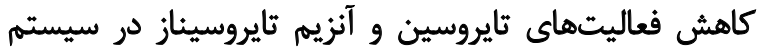

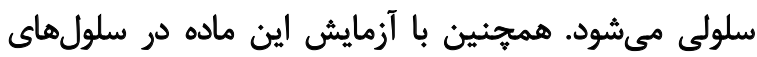

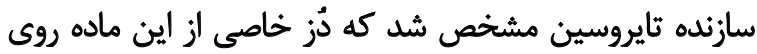

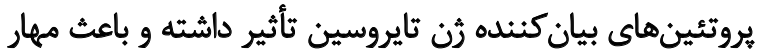

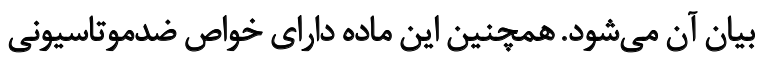

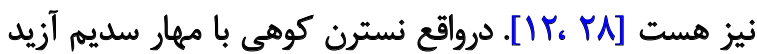

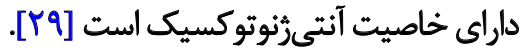

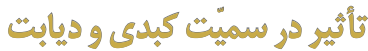

مطالعه اثر سميت كبدى با تتراكلرايد كربن، افزايش سطح

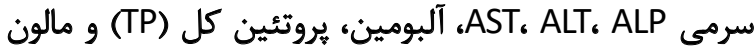
دى آلدئيد (MDA) رانشان داد. اين در حالى بود كه يس از درمان

6. Beta-sitosterol
ميدهند كه عصاره نسترن كوهى به دليل داشتن فعاليتهاي

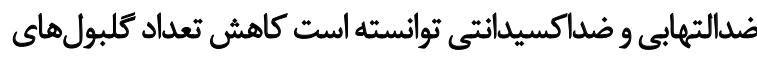

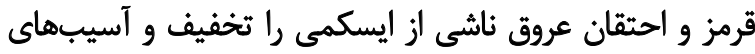

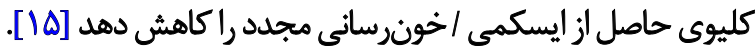

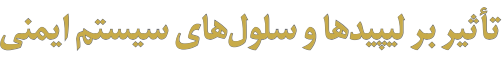

نسترن كوهى حاوى آلفالينولنيك اسيد است ازئ آنجا كه اسيد

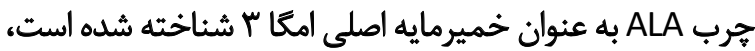

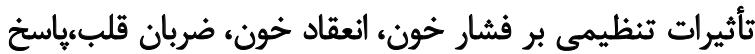

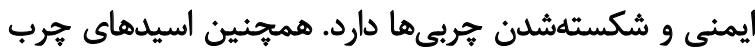

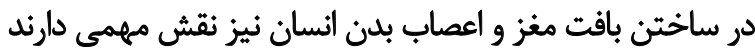

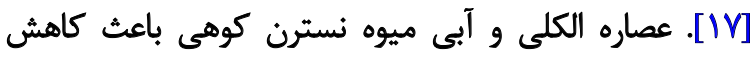

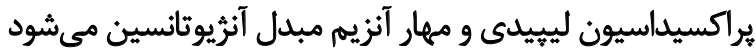

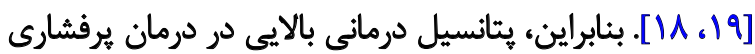

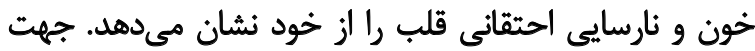

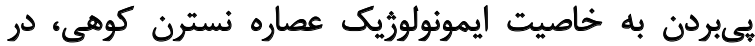

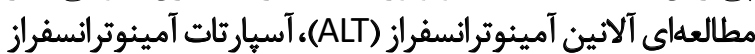
(AST)

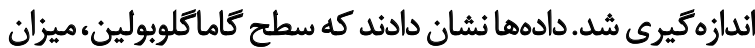

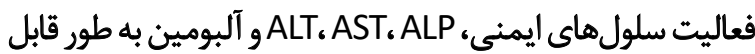

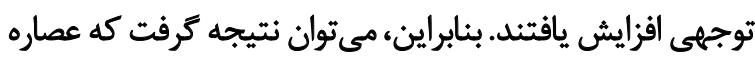

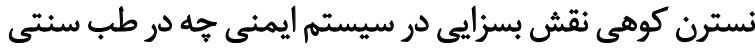

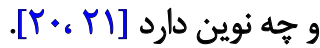

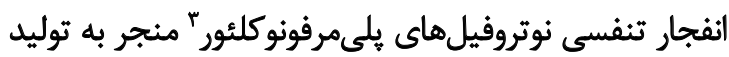

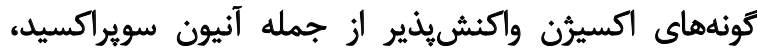

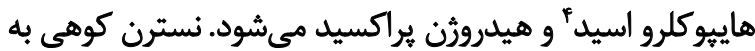

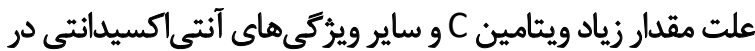

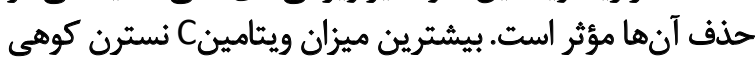

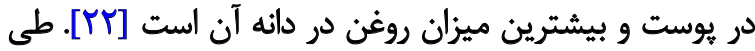

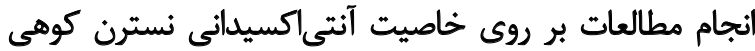

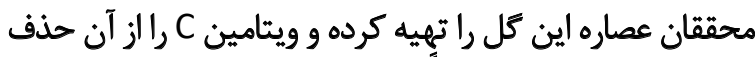

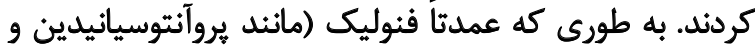

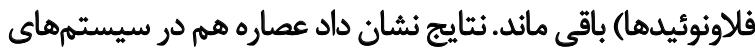

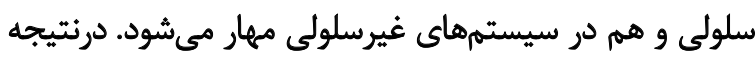

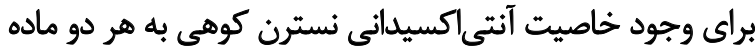
ويتامين C و يلى فنوليك نياز است [Tr]

آزمايشها بر روى اثرات ايمنى نسترن كوهى نشان مى دهد

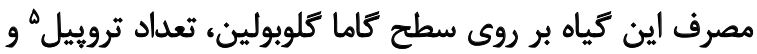
مونوسيت و فاكوسيتوز اثر داشته و باعث افزايش آنها ماني مئشود. ترويل
2. Alpha linolenic acid
3. Polymerphonuclear neutrophils
4. Hypochlorous Acid (HOCl)
5. Tropyl 


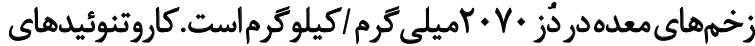

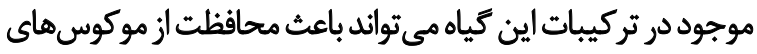

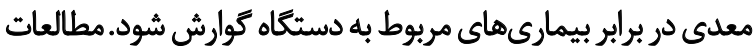

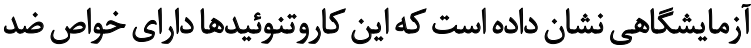

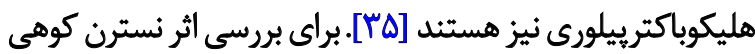

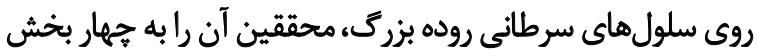

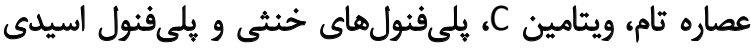

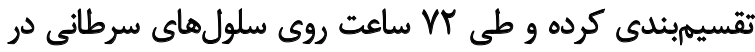

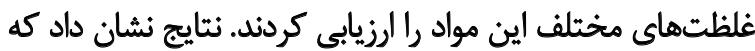

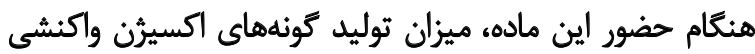

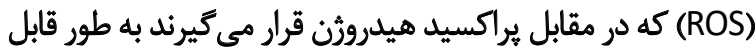

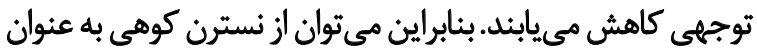

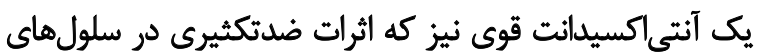

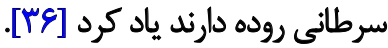

\section{اثر هر بيماري لوسمي}

لوسمى ميلوئيد حاد` نوعى سرطان خون است كه علاتم

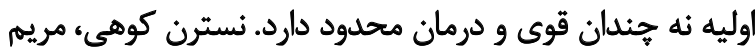

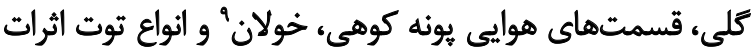

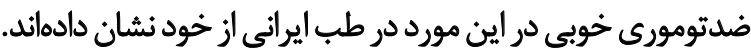

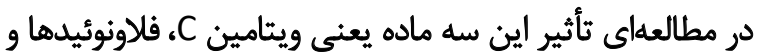

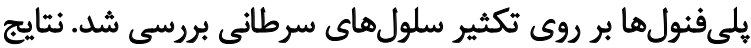

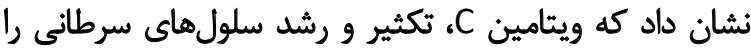

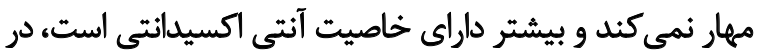

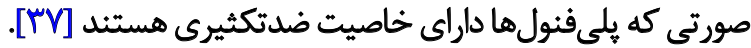
طى مطالعاتى اثثر آنتى لوسميك عصاره آبى و الكلى اين گياهان

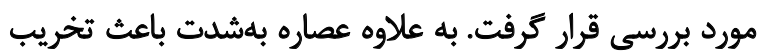

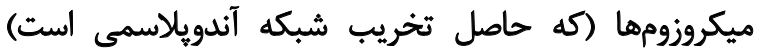

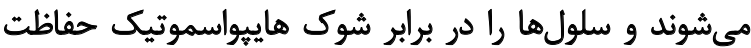

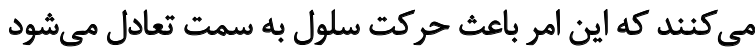

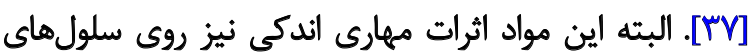

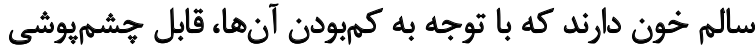

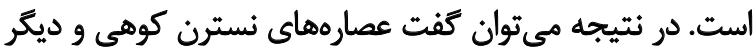

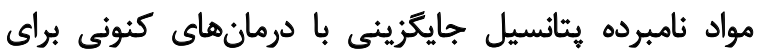

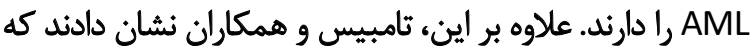

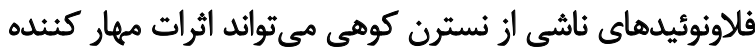

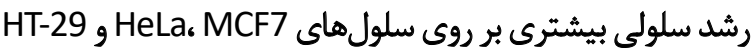

نسبت به ساير مشتقات اين كياه داشته باشند [برى]

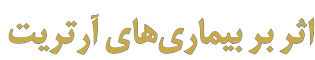

عصاره نسترن كوهى داراى خاصيت ضدالتهابى و آنابوليكى در

8. Acute Myeloid Leukemia (AML)

9. Hippophae rhamnoides

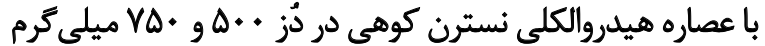

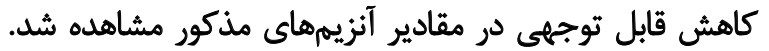

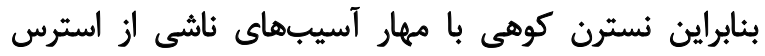

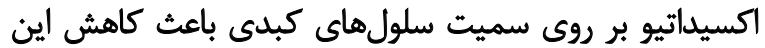

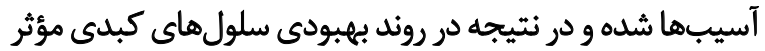

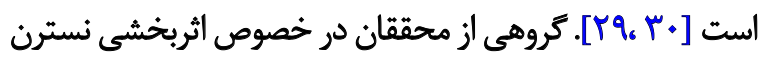

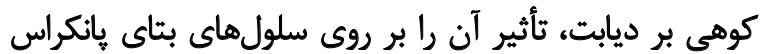

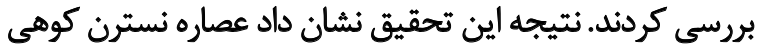

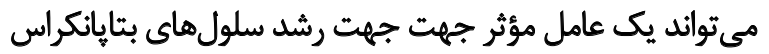

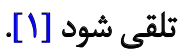

نتايج در مطالعه ديكر نشان داد مكمل عصاره كياه نسترن

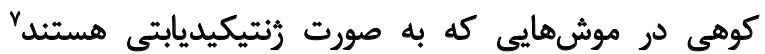

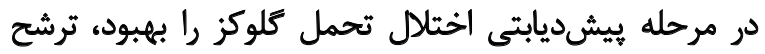

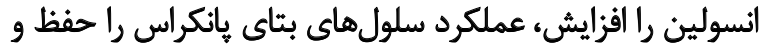

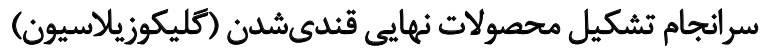

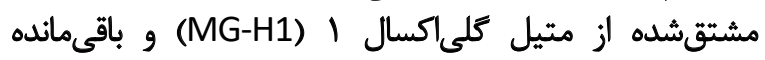
كربوكسيمتيل لايزين راسركوب كرده است [است]

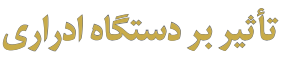

كتايج حاصل از مطالعات انجامشده در مورد ثأثيرات نسترن

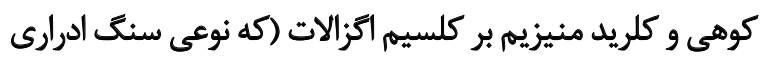

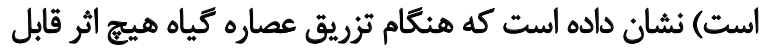

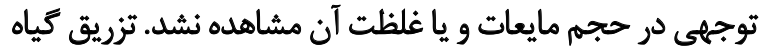

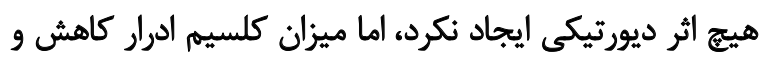

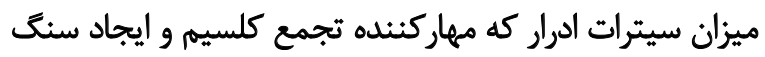
است، افزايش يافت سيترات آتران

هنغام تزريق كلريد منيزيم برعكس همين رخداد اتفات اتفاق افتاد

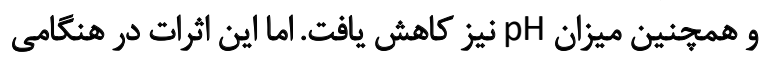

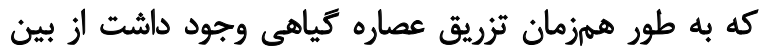

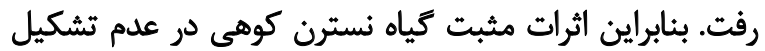

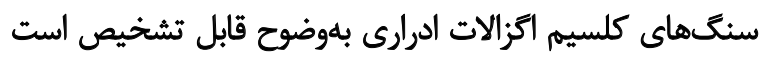

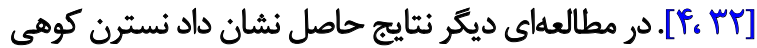

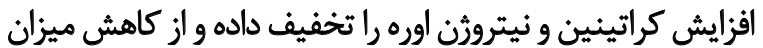

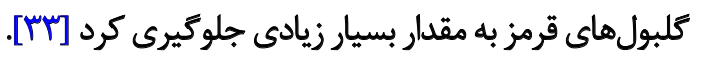

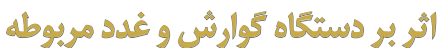

نتايج مطالعات انجامشده درباره عصاره هيدروالكلى نسترن

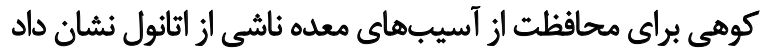

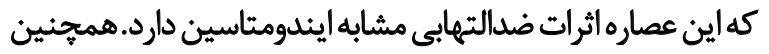

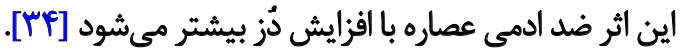
برخى از مطالعات نشان دادند ميوه اين كياه داراى خواص ضد

7. Spontaneously Diabetic Torii (SDT) 
نيز بيشتر از تركيب آن با تموزولوميد و يا تموزولوميد جداكانه

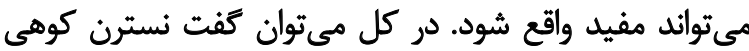

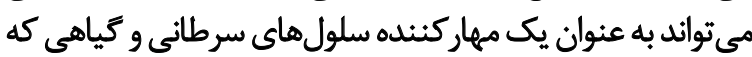

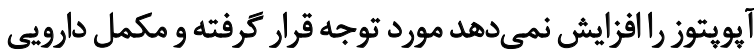

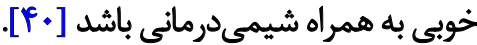

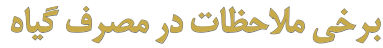

على غومه نتايج مثبت مصرف كياه نسترن كوهى كه ناشى أز مواد

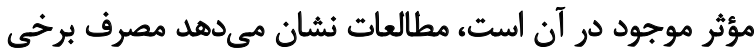

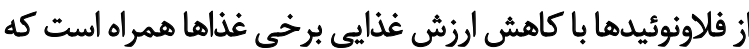

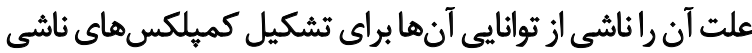

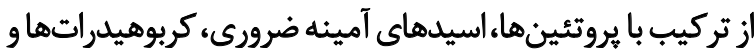

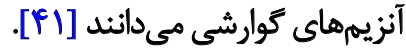

مصرف خوراكى ميوه كل سرخ هيج كونه مسموميتى ايجاد

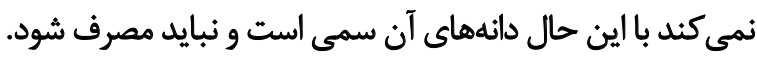

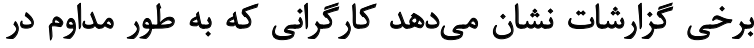

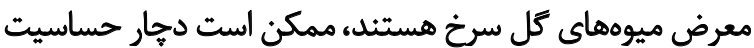

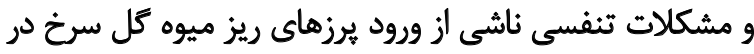

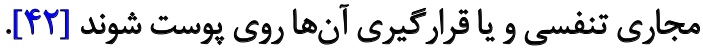

\section{تتيجلكَيرى}

نتايج حاصل از اين مقاله مرورى نشان مىدهد كياه نسترن

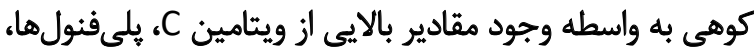

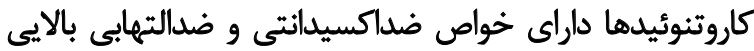

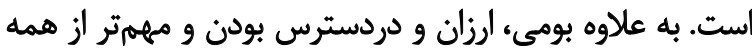

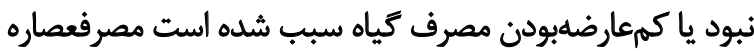

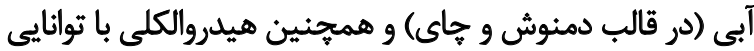

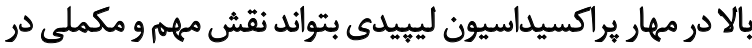

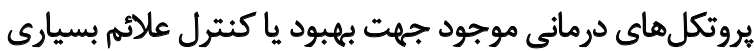

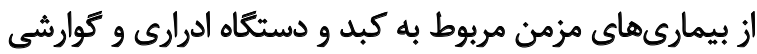

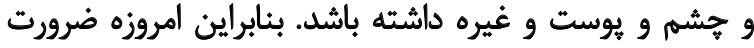

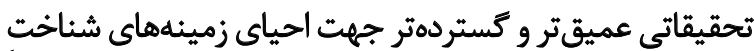

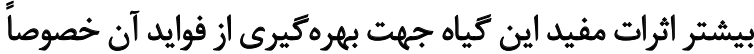
در جنبههاى دارويى احساس مىشود.

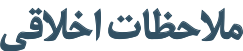

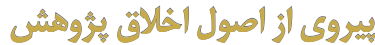

در نكارش مقاله اصول اخلاق نشر رعايت شده است.

$$
\text { Ullo }
$$

اين مقاله حامى مالى نداشته است.
سلولهاى كندروسيت است. يكى ديكر از موارد استفاده نسترن

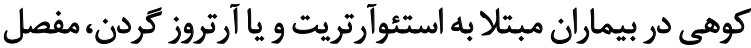

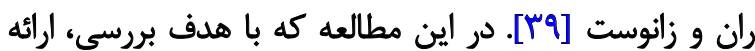

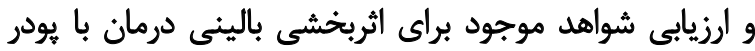

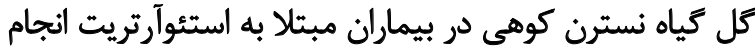

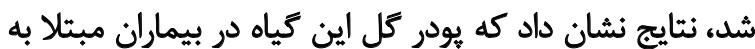

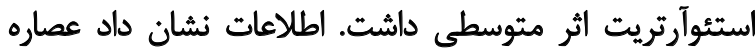

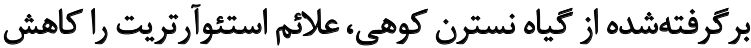

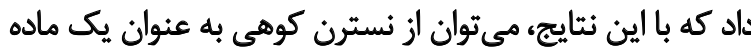

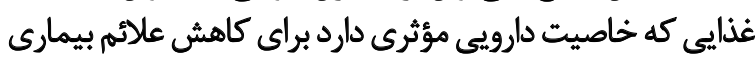
استئوآرتريت استفاده كرد.

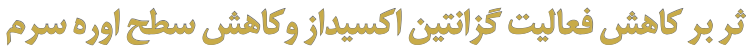

ميوه كياه نسترن كوهى به طور سنتى به منظور درمان

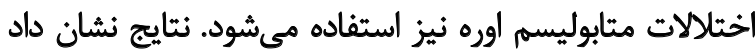

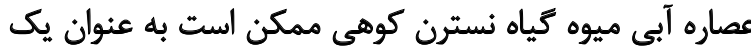

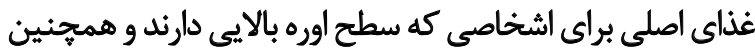

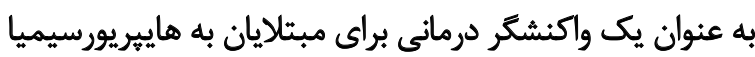

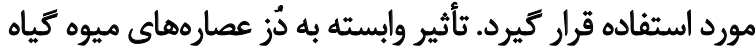

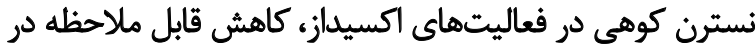

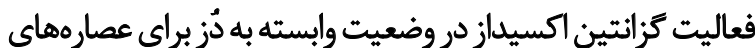

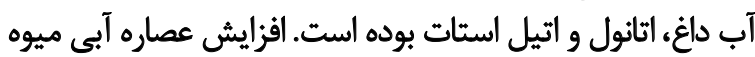

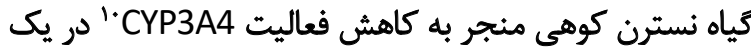

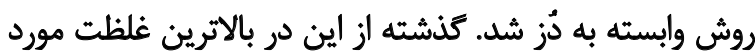

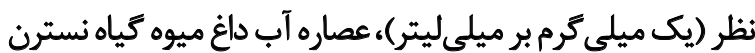

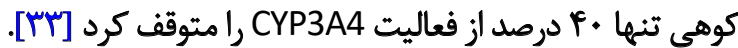

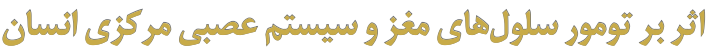

طبق مطالعاتى تأثير عصاره نسترن كوهى بر سه رده سلولى از

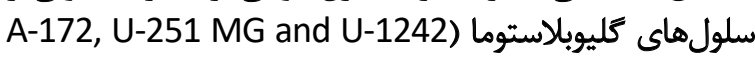
(MG cell lines

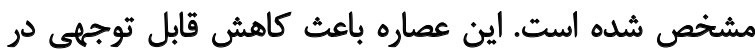

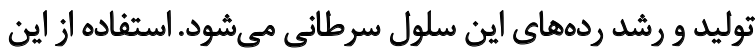

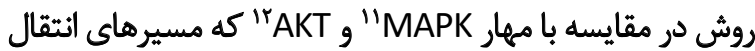

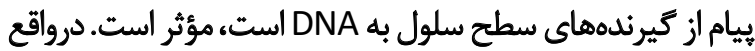

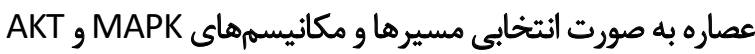

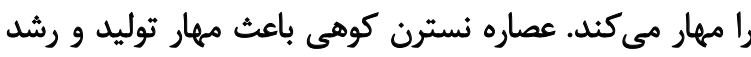

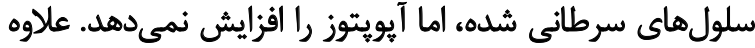

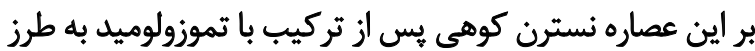

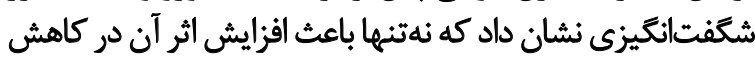

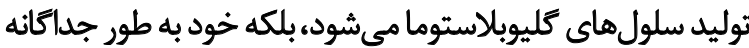

10. Cytochrome P450 3A4

11. Mitogen-activated protein kinase

12. Threonine-protein kinase 


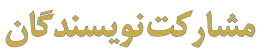

ايدهيردازي و اصلاح مقاله:على زارعى، سعيد جنكيزي آشتيانى

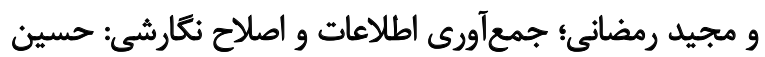

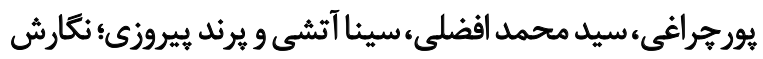

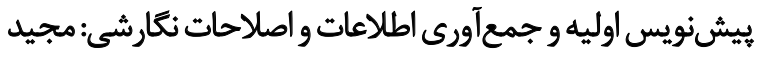

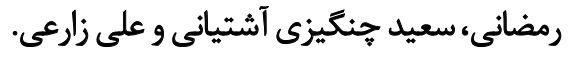

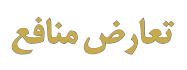

بنابر اظهار نويسندكان اين مقاله تعارض منافع ندارد.

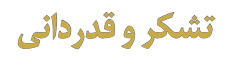

بدينوسيله از مساعدت معاونت محترم تحقيقات و فناورى

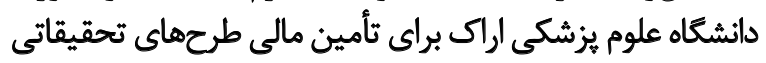
مذكور صميمانه تشكر مىشود. 


\section{References}

[1] Fattahi A, Niyazi F, Shahbazi B, Farzaei MH, Bahrami G. Antidiabetic mechanisms of rosa canina fruits: An in vitro evaluation. J Evid Based Complementary Altern Med. 2017; 22(1):127-33. [DOI:10.1177/2156587216655263] [PMID] [PMCID]

[2] Javanmard M, Asadi-Gharneh HA, Nikneshan P. Characterization of biochemical traits of dog rose (Rosa canina L.) ecotypes in the central part of Iran. Nat Prod Res. 2018; 32(14):1738-43. [DOI:10.1080/14786419.2 017.1396591] [PMID]

[3] Brasovan A, Mândroc V, Câmpean R, Petean I, Codrea V, Arghir G. Calcium and magnesium content in brier (Rosa canina $L$.) fruits at the. Analele Universitatii din Oradea: Fascicula Biologie. 2018; 11(1):5-9.

[4] Tayefi-Nasrabadi H, Sadigh-Eteghad S, Aghdam Z. The effects of the hydroalcohol extract of Rosa canina $L$. fruit on experimentally nephrolithiasic Wistar rats. Phytother Res. 2012; 6(1):78-85. [DOI:10.1002/ ptr.3519] [PMID]

[5] Heinemann W. The older pliny, natural history. Cambridge: Harvard University Press; 1966.

[6] Sharafkandi A. [The Persian translation of qanoun fi al-tibb (or the canon of medicine) Vol. 2 (Persian)]. Tehran: Soroush Press; 2008.

[7] Selahvarzian A, Alizadeh A, Amanolahi Baharvand P, Eldahshan AO, Rasoulian B. Medicinal properties of Rosa canina L. Herb Med J. 2018; 2(4):77-84.

[8] Živković J, Stojković D, Petrović J, Zdunić G, Glamočlija J, Soković M. Rosa canina $L$, new possibilities for an old medicinal herb. Food Funct. 2015; 6(12):3687-92. [DOI:10.1039/C5FO00820D] [PMID]

[9] Paunovic D, Kalusevic A, Petrovic T, Urosevic T, Djinovic D, Nedovic V, et al. Assessment of chemical and antioxidant properties of fresh and dried rosehip (Rosa canina L.). Notulae Botanicae Horti Agrobotanici Cluj-Napoca. 2018; 47(1):34-45. [DOI:10.15835/nbha47111221]

[10] Taneva I, Petkova N, Dimov I, Ivanov I, Denev P. Characterization of Rose hip (Rosa canina L.) Fruits extracts and evaluation of their in vitro antioxidant activity. J Pharmacogn Phytochem. 2016; 5(2):35-38.

[11] Kilicgun H, Altiner D. Correlation between antioxidant effect mechanisms and polyphenol content of Rosa canina. Pharm Mag. 2010; 6(23):238-41. [DOI:10.4103/0973-1296.66943] [PMID] [PMCID]

[12] Jemaa HB, Jemia AB, Khlifi S, Ahmed HB, Slama FB, Benzarti A, et al. Antioxidant activity and $\alpha$-amylase inhibitory potential of Rosa canina L. African J Tradition, Complementary Altern Med. 2017; 14(2):1-8. [DOI:10.21010/ajtcam.v14i2.1] [PMID] [PMCID]

[13] Nojavan S, Khalilian F, Kiaie FM, Rahimi A, Arabanian A, Chalavi S. Extraction and quantitative determination of ascorbic acid during different maturity stages of Rosa canina L. fruit. J Food composit Anal. 2008; 21(4):300-5. [DOI:10.1016/j.jfca.2007.11.007]

[14] Rezaei M, Zamani S, Ghasemi A, Rahimi A, Nabi N, Changizi- Ashtiyani S,et al. The Effects of hydroalcoholic extract of melissa officinalis. I on the level of renal function and liver enzymes in diabetic rats. Iran J EndocrinolMetabol. 2016; 17(5):353-61.

[15] Changizi-Ashtiyani S, Najafi H, Jalalvandi S, Hosseinei F. Protective effects of Rosa canina $L$ fruit extracts on renal disturbances induced by reperfusion injury in rats. Iran J Kid Diseas. 2013; 7(4):290-8.

[16] Changizi- Ashtiyani S, Najafi H, Kabirinia K, Jamebozorky L. The effect of oral omega-3 administration on the reduction of renal dysfunction induced by ischemia/reperfusion in rats. Irab J Kidney Dis. 2012; 6(4):275-83.
[17] Taghizadeh M, Rashidi AA, Taherian AA, Vakili Z, Sajad Sajadian M, Ghardashi M. Antidiabetic and antihyperlipidemic effects of ethanol extract of Rosa canina L. fruit on diabetic rats: An experimental study with histopathological evaluations. J evid Compl Alter Med. 2016; 21(4):25-30. [DOI:10.1177/2156587215612626] [PMID]

[18] Georgieva, Silviya, George Angelov, Stanislava Boyadzhieva. Concentation of vitamin $\mathrm{C}$ and antioxidant activity of rosehip extracts. J Chem Technol Metal. 2014; 49(5):451-4.

[19] Ahmad N, Anwar F, Gilani AU. Rose Hip (Rosa canina L.) oils. In: Preedy, VR. Essential oils in food preservation, flavor and safety. Cambridge: Academic Press. 2016; 23(11):667-75. [DOI:10.1016/B978-0-12-4166417.00076-6]

[20] Sadigh-Eteghad S, Tayefi-Nasrabadi H, Aghdam Z, Zarredar H, Shanehbandi D, Khayyat L, et al. Rosa canina L. Fruit hydro-alcoholic extract effects on some immunological and biochemical parameters in rats. Bioimpacts. 2011; 1(4):219-24.

[21] Changizi-Ashtiyani S, Berenji S, Zarei A, Ramezani M, Hosseini N. The effects of the extract of Rosa canina $L$. on lipid profiles, liver and thyroid functions in hypercholesterolemic rats. JKUM. 2018; 25(4):318-27.

[22] Jafarirad S, Mehrabi M, Divband B, Kosari-Nasab M. Biofabrication of zinc oxide nanoparticles using fruit extract of Rosa canina and their toxic potential against bacteria: A mechanistic approach. Mater SciEngineer. 2016; 59(11):296-302. [DOI:10.1016/j.msec.2015.09.089] [PMID]

[23] Fromm M, Bayha S, Kammerer DR, Carle R. Identification and quantitation of carotenoids and tocopherols in seed oils recovered from different Rosaceae species. J Agric Food Chem. 2012; 60(43):10733-42. [DOI:10.1021/jf3028446] [PMID]

[24] Özdemir E, Alpınar K. An ethnobotanical survey of medicinal plants in western part of central Taurus Mountains: Aladaglar (Nigde-Turkey). J Ethno Pharm. 2015; 166(3):53-65. [DOI:10.1016/j.jep.2015.02.052] [PMID]

[25] Larsen E, Kharazmi A, Christensen LP, Christensen SB. An antiinflammatory galactolipid from Rose hip (rosa $c$ anina) that inhibits chemotaxis of human peripheral blood neutrophils in vitro. J Natur Prod. 2003 66(7):994-5. [DOI:10.1021/np0300636] [PMID]

[26] Al-Yafeai A,Böhm V. In vitro bioaccessibility of carotenoids and vita$\min E$ in rosehip products and tomato paste as affected by pectin contents and food processing. J Agric Food Chem. 2018; 66(15):3801-9. [DOI:10.2147/BTAT.S91385]

[27] Ilbay Z, Sahin S, Kirbaslar SI. Investigation of polyphenolic content of rose hip (Rosa canina L.) tea extracts: A comparative study. Foods. 2013 2(1):43-52. [DOI:10.3390/foods2010043] [PMID] [PMCID]

[28] Jiménez S, Gascón S, Luquin A, Laguna M, Ancin-Azpilicueta C, Rodríguez-Yoldi (eCollection). Rosa canina extracts have antiproliferative and antioxidant effects on caco-2 human colon cancer. PLoS One. 2016 11(7):1-11. [DOI:10.1371/journal.pone.0159136] [PMID] [PMCID]

[29] Chrubasik C, Roufogalis BD, Müller-Ladner U, Chrubasik S. A systematic review on the Rosa canina effect and efficacy profiles. Phytother Res. 2008; 22(6):725-33. [DOI:10.1002/ptr.2400] [PMID]

[30] Sadeghi H, Hosseinzadeh S, Touri MA, Ghavamzadeh M, Barmak MJ. Hepatoprotective effect of Rosa canina fruit extract against carbon tetrachloride induced hepatotoxicity in rat. Avicenna J Phytomed. 2016 6(2):181-8.

[31] Chen SJ, Aikawa C, Yoshida R, Kawaguchi T, Matsui T. Anti-prediabetes effect of rose hip (Rosa canina) extract in spontaneously diabetic Torii rats. J Sci Food Agric. 2017; 97(12):3923-8. [DOI:10.1002/ jsfa.8254] [PMID] 
[32] Grases F, Masárová L, Costa-Bauzá A, March JG, Prieto R, Tur JA. Effect of "Rosa Canina" infusion and magnesium on the urinary risk factors of calcium oxalate urolithiasis. Planta Med. 1992; 58(6):509-12. [DOI:10.1055/s-2006-961537] [PMID]

[33] Kikuchi H, Kogure S, Arai R, Saino K, Ohkubo A, Tsuda T, et al. Rosehip inhibits xanthine oxidase activity and reduces serum urate levels in a mouse model of hyperuricemia. Biomed Rep. 2017; 6(5):539-44. [DOI:10.3892/br.2017.888] [PMID] [PMCID]

[34] Lattanzio F, Greco E, Carretta D, Cervellati R, Govoni P, Speroni E. In vivo anti-inflammatory effect of Rosa canina L. extract. J Ethnopharmacol. 2011; 137(1):880-5. [DOI:10.1016/j.jep.2011.07.006] [PMID]

[35] Ayati Z, Amiri MS, Ramezani M, Delshad E, Sahebkar A, Emami SA Phytochemistry, traditional uses and pharmacological profile of rose hip. Curr Pharm Des. 2018; 24(35):4101-24. [DOI:10.2174/138161282 4666181010151849] [PMID]

[36] Jiménez S, Gascón S, Luquin A, Laguna M, Ancin-Azpilicueta C, Rodríguez-Yoldi MJ. Rosa canina extracts have antiproliferative and antioxidant effects on caco-2 human colon cancer. PLoS One. 2016; 11(7):1-14. [DOI:10.1371/journal.pone.0159136] [PMID] [PMCID]

[37] Zhamanbayeva GT, Aralbayeva AN, Murzakhmetova MK, Tuleukhanov ST, Danilenko M. Cooperative antiproliferative and differentiationenhancing activity of medicinal plant extracts in acute myeloid leukemia cells. Biomed Pharmacother. 2016; 13(82):80-9. [DOI:10.1016/j. biopha.2016.04.062] [PMID]

[38] Tumbas VT, Ĉanadanović-Brunet JM, Ĉetojević-Simin DD, Ćetković GS, Đilas SM, Gille L. Effect of rosehip (Rosa canina L.) phytochemicals on stable free radicals and human cancer cells. J Sci Food Agric. 2012; 92(6):1273-81. [DOI:10.1002/jsfa.4695] [PMID]

[39] Şahan A, Duman S, Çolak SÖ, Çinar E, Bilgin R. Determination of some hematological and non-specific immune defences, oxidative stress and histopathological status in rainbow trout (oncorhynchus mykiss) fed Rosehip (Rosa canina) to yersinia ruckeri. Turkish JFish Aquat Sci. 2017; 17(1):91-100. [DOI:10.4194/1303-2712-v17_1_11]

[40] Cagle P, Idassi O, Carpenter J, Minor R, Goktepe I, Martin P. Effect of Rosehip (Rosa canina) extracts on human brain tumor cell proliferation and apoptosis. Ournal Cancer Ther. 2012; 3(5):534-5. [DOI:10.4236/jct.2012.35069]

[41] Winther K, Vinther Hansen AS, Campbell-Tofte J. Bioactive ingredients of Rose hips (Rosa canina $L$ ) with special reference to antioxidative and anti-inflammatory properties. Botanics. 2016; 6:11-23. [DOI:10.2147/BTAT.S91385]

[42] Momeni M, [Nutrient rich in vitamin C (Persian)] [Internet]. 2018 [Updated 2018 May 11]. Available from: http://agronic.blogfa.com/post/44 